03

\title{
Кинетика примеси ионов в собственном газе во внешнем гармоническом электрическом поле
}

\author{
() А.Я. Эндер, ${ }^{1}$ И.А. Эндер, ${ }^{2}$ А.Б. Герасименко ${ }^{1,9}$ \\ ${ }^{1}$ Физико-технический институт им. А.Ф. Иофрфе РАН, \\ 194021 Санкт-Петербург, Россия \\ ${ }^{2}$ Санкт-Петербургский государственный университет, \\ 198504 Санкт-Петербург, Россия \\ ฯ e-mail: gerasimenko.alexander@mail.ioffe.ru
}

(Поступило в Редакцию 24 февраля 2016 г.)

Рассмотрена пространственно-однородная задача о поведении примеси ионов в фоновом газе после включения гармонического электрического поля с произвольными параметрами для различных законов взаимодействия частиц. Уравнение Больцмана решено модифицированным моментным методом. Изучены ионная функция распределения и ее первые моменты. Показано, что универсальные аналитические выражения для плотности тока и энергии ионов, полученные нами ранее в случае малого отношения амплитуды поля к его частоте, имеют значительно более широкую область применения.

\section{1. Введение}

Теоретическое исследование движения малой примеси заряженных частиц в различных газах при наличии электрических полей проводилось, начиная с середины двадцатого века [1-3]. Особенно активно оно развивалось на протяжении последних 40 лет. Актуальность исследований обусловлена получением различных экспериментальных результатов (экспериметны с дрейфовыми трубками и разрядами), которые требовали надежного теоретического описания [4], а также развитием различных технологий (плазменная обработка материалов, спектрометрия ионной подвижности, разработка детекторов излучения) $[5,6]$.

Если кинетический подход к описанию движения электронов достаточно развит $[7,8]$, то для ионов применяют либо упрощенные модели уравнения Больцмана [9], либо численное моделирование методом Монте-Карло [10]. Эти подходы позволяют весьма приближенно исследовать физические моменты и не дают надежных сведений о функции распределения, особенно в области больших скоростей.

Одним из наиболее продуктивных методов кинетического описания оказался моментный метод решения уравнения Больцмана и его модификации $[11,12]$. Этот метод заключается в разложении функции распределения (ФР) ионов по системе ортогональных полиномов и последующем преобразовании уравнения Больцмана к системе линейных дифференциальных уравнений. Основной сложностью при применении моментного метода является расчет матричных элементов (МЭ) интеграла столкновений. В известных нам работах в основном рассматриваются либо малое число членов разложения ФР [13], либо случай, когда отношение масс иона к массе атома фонового газа невелико. Например, метод расчета интеграла столкновений, предложенный Кумаром [14], оказывается эффективным только при значениях отношения масс, не превышающих 0.1 [15]. Провести расчеты для произвольных (в том числе и для равных) масс ионов и атомов стало возможным после получения рекуррентных соотношений для нелинейных МЭ $[16,17]$. Это позволило строить матричные элементы со сколь угодно большими индексами. Однако, как показано в [18], построенный нами в [16] однотемпературный моментный метод при сильных полях расходится. В $[18,19]$ для улучшения сходимости был использован модифицированный моментный метод, который получил дальнейшее развитие в настоящей работе.

Настоящая работа является продолжением работы [20], где изучалась эволюция примеси ионов в собственном газе в переменном высокочастотном электрическом поле при условии малости отношения амплитуды поля к его частоте. Рассматривались различные законы взаимодействия ионов с атомами. Использовалась введенная нами ранее специальная нормировка, в которой безразмерная подвижность в слабом постоянном электрическом поле равна единице. Показано, что в этой нормировке зависимости от времени плотности электрического тока и приобретаемой ионами полной энергии становятся универсальными и не зависят от сечения взаимодействия.

В настоящей работе рассматриваются гармонические поля произвольной амплитуды и частоты. Применяется модифицированный моментный метод с температурой базиса, определенным образом зависящей от времени, и особым способом построения модифицированных линейных МЭ. В большом диапазоне изменения параметров поля нами изучены эволюция функции распределения, а также зависимости плотности тока и энергии ионов от времени. Показано, что универсальные аналитические формулы для тока и энергии, полученные в [20], оказались справедливыми в значительно большей области изменения параметров поля. Определены области изменения этих параметров, что может оказаться полезным при проведении экспериментов. 
Таблица 1. Коэффициент перехода от $\tau$ к $\beta$-нормировке для различных моделей взаимодействия

\begin{tabular}{c|c|c|c|c|c|c}
\hline Модель & СЕМ & РМM & CEHS & HS & MM & ANHS \\
\hline$\beta$ & 1 & 2 & 0.4826 & 0.9578 & 0.4547 & 2.8665
\end{tabular}

\section{Постановка задачи}

Как и в [20], рассматривается пространственно однородная задача об эволюции примеси ионов в фоновом газе при включении внешнего гармонического электрического поля. Массы ионов и атомов считаем равными. Предполагается, что примесь не влияет на фоновый газ, а взаимодействие между частицами примеси пренебрежимо мало. Функцию распределения фонового газа $f_{a}(\mathbf{v})$ по скоростям считаем максвелловской. Функция распределение ионов $f(\mathbf{v})$ в начальный момент времени также имеет максвелловское распределение с температурой атомов $T_{0}$. Все процессы взаимодействия ионов с атомами считаем упругими. Внешнее электрическое поле направлено по оси $z$.

Переходя к безразмерным переменным в уравнении Больцмана, в качестве единицы измерения времени выберем $\tau_{\beta}=\beta \tau$. Здесь $\tau-$ среднее время между столкновениями для конкретной модели взаимодействия, а $\beta-$ безразмерная подвижность, вычисленная в стандартной $\tau$-нормировке в постоянном слабом электрическом поле. $\mathrm{B}$ табл. 1 приведены значения $\beta$ для всех рассматриваемых нами моделей взаимодействия.

В качестве единицы измерения скорости выберем тепловую скорость $v_{T}=\sqrt{2 k T_{0} / m}$. Тогда безразмерные напряженность электрического поля и его частота определяются следующим образом

$$
\epsilon(\tilde{t})=\frac{e E_{0} \tau_{\beta} \cos (\Omega t)}{m v_{T}}=\epsilon_{0} \cos (\omega \tilde{t}), \quad \omega=\Omega \tau_{\beta}, \quad \tilde{t}=t / \tau_{\beta}
$$

Здесь $E_{0}$ и $\Omega$ - размерные амплитуда и частота внешнего электрического поля, $e-$ величина заряда электрона, $m$ - масса иона, а $\epsilon_{0}-$ безразмерная амплитуда внешнего поля.

Уравнение Больцмана в безразмерных переменных принимает вид

$$
\frac{\partial f(\mathbf{c}, \tilde{t})}{\partial \tilde{t}}+\epsilon(\tilde{t}) \frac{\partial f(\mathbf{c}, \tilde{t})}{\partial \mathbf{c}}=\hat{I}(f(\mathbf{c}, \tilde{t}), M(\mathbf{c})),
$$

где

$$
M(\mathbf{c})=\left(\frac{1}{\pi}\right)^{3 / 2} \exp \left(-\mathbf{c}^{2}\right), \quad \mathbf{c}=\frac{\mathbf{v}}{v_{T}},
$$

a $\hat{I}(f(\mathbf{c}, \tilde{t}), M(\mathbf{c}))$ - линейный интеграл столкновений.

В настоящей работе будут рассмотрены сечения взаимодействия, соответствующие степенным, „псевдостепенным“ и „квазистепенным“ потенциалам.
Для степенных потенциалов $\left(V \sim \frac{1}{r^{v}}\right)$ дифференциальное сечение рассеяния $\sigma(g, z)$ представляется в виде

$$
\begin{gathered}
\bar{g} \sigma(\bar{g}, z)=\sigma_{0} \bar{g}^{2 \mu} F(z), \\
\bar{g}=g / v_{T}, \quad z=\sin ^{2}(\theta / 2), \quad \mu=(v-4) /(2 v) .
\end{gathered}
$$

Здесь $g$ - модуль относительной скорости сталкивающихся частиц, $\theta-$ угол рассеяния, $\sigma_{0}-$ некоторая эффективная площадь взаимодействия [21]. Величина $F(z)$ - это угловая часть сечения, полностью определяемая параметром v. Модель „псевдостепенных потенциалов" - это модель, в которой рассеяние по углам предполагается изотропным, а зависимость сечения рассеяния от модуля скорости - такой же, как у степенного потенциала. Можно определить множество моделей „квазистепенных потенциалов“ с фиксированным значением $v$, для которых сохраняется зависимость $\sigma$ от $g$ как для степенного потенциала, а угловая зависимость предполагается произвольной.

В работе рассмотрены $\tau$ - модели (модели с постоянным временем между столкновениями): CEM, ММ и РММ и $\lambda$-модели (модели с постоянной длиной свободного пробега): HS, CEHS и ANHS:

1. CEM и CEHS имеют угловую часть сечения рассеяния, соответствующую резонансной перезарядке (рассеяние на 180。); для СЕМ-модели $(\mu=0)$ полное сечение рассеяния обратно пропорционально относительной скорости, а для CEHS-модели $(\mu=0.5)$ сечение не зависит от скорости.

2. ММ и РММ - максвелловские и псевдомаксвелловские молекулы $(\mu=0)$; полное сечение рассеяния обратно пропорционально относительной скорости. Для PMM - распределение по углам изотропно.

3 . HS и ANHS $(\mu=0.5)$ : полное сечение не зависит от скорости; для HS-модели рассеяние по углам изотропно, а для ANHS-модели сечение пропорционально $\cos ^{2}(\theta / 2)=1-z$.

В [20] рассматривалась аналогичная постановка задачи в случае высоких частот при условии малости параметра $\alpha$, характеризующего переменное гармоническое поле

$$
\alpha=\frac{E_{0} e}{m \Omega v_{T}}=\frac{\epsilon_{0}}{\omega} .
$$

В настоящей работе мы рассматриваем любые $\alpha$, что соответствует произвольным отклонениям ФР от равновесия. Характерной чертой параметра $\alpha$ является его независимость от выбора временно́го масштаба.

\section{Модифицированный моментный метод}

В [20] расчеты ФР и ее моментов проводились нестационарным стандартным (однотемпературным) моментным методом с предложенным нами ранее способом расчета МЭ интеграла столкновений [16]. При малых $\alpha$ такой метод сходился. При переходе к сильным полям стандартный моментный метод даже с использованием 
большого числа МЭ оказывается непригодным. Происходит очень быстрый рост моментов, и метод расходится. Связано это с тем, что температура ионов при развитии процесса может намного превышать температуру фонового газа.

В настоящей работе мы рассматриваем новый метод расчета линейных МЭ в случае, когда температура базиса не равна температуре атомов. В этом случае приходится использовать подмножество нелинейных однотемпературных МЭ. Такие МЭ всегда вычисляются по ходу расчета однотемпературных линейных МЭ с помощью предложенной нами рекуррентной процедуры $[16,18]$. Кроме того, мы используем нестационарный метод, полагая температуру базиса, определенным образом зависящей от времени.

Вопрос о расчете линейных МЭ в двухтемпературном методе обсуждался в литературе [12,15]. Однако в этих работах построение МЭ в новом базисе проводилось путем вычисления сложных интегралов и приводило к большим затратам машинного времени при не всегда удовлетворительной точности. Переход к базису с переменной температурой предложен в работе [15], однако рассмотренный там метод расчета МЭ не позволяет вычислять МЭ с большими индексами при равных массах атомов и ионов. Кроме того, проблематичным является расчет ФР в области достаточно больших скоростей.

Итак, представим ФР ионов в виде

$$
f(\mathbf{c}, \tilde{t})=M\left(c_{1}\right) \sum_{l=0}^{L_{0}} \sum_{r_{1}=N_{0}-l}^{N_{0}} \bar{C}_{r_{1}, l}(\tilde{t}) H_{r_{1}, l}\left(\mathbf{c}_{1}\right), \quad L_{0} \leq N_{0},
$$

$$
H_{r_{1}, l}\left(\mathbf{c}_{1}\right)=P_{l}(\cos \Theta) c_{1}^{l} S_{l+1 / 2}^{r_{1}}\left(c_{1}^{2}\right), \quad \mathbf{c}_{1}=\sqrt{\frac{m}{2 k T_{1}}} \mathbf{v} .
$$

Здесь $M\left(c_{1}\right)=(1 / \pi)^{3 / 2} \exp \left(-c_{1}^{2}\right)-$ весовой максвеллиан, $P_{l}(\cos \Theta)$ - полиномы Лежандра, а $S_{l+1 / 2}^{(r)}\left(c_{1}^{2}\right)-$ полиномы Сонина (Лагерра).

Система безразмерных моментных уравнений, соответствующая уравнению Больцмана (2), имеет вид

$$
\begin{gathered}
\frac{\partial \bar{C}_{r_{1}, l}}{\partial \tilde{t}}+\epsilon(\tilde{t}) \sqrt{R}\left(\frac{2 r_{1}(l+1)}{2 l+3} \bar{C}_{r_{1}-1, l+1}-\frac{2 l}{2 l-1} \bar{C}_{r_{1}, l-1}\right) \\
=\sum_{r=N_{0}-l}^{. N_{0},} \bar{\Lambda}_{r_{1}, r, l} \bar{C}_{r_{1}, l}, \quad l \leq L_{0}, \quad R=T_{0} / T_{1} .
\end{gathered}
$$

Линейные матричные элементы интеграла столкновений $\bar{\Lambda}_{r_{1}, r, l}$ определяются следующим образом:

$$
\begin{gathered}
\bar{\Lambda}_{r_{1}, r, l}=\int H_{r_{1}, l}\left(\mathbf{c}_{1}\right) \hat{I}\left(M\left(c_{1}\right) H_{r, l}\left(\mathbf{c}_{1}\right), M(c)\right) d \mathbf{c}_{1} / g_{r_{l}, l}, \\
g_{r, l}=\frac{(2 r+2 l+1) ! !}{(2 r) ! ! 2^{l}(2 l+1)} .
\end{gathered}
$$

Разложим максвеллиан $M(c)$ по новому базису

$$
M(c)=R^{3 / 2} M\left(c_{1}\right) \sum_{k}(1-R)^{k} S_{1 / 2}^{k}\left(c_{1}^{2}\right) .
$$

Тогда для МЭ $\bar{\Lambda}_{r_{1}, r, l}$ в новом базисе при несовпадении температур $T_{1}$ и $T_{0}$ получим следующее выражение

$$
\bar{\Lambda}_{r_{1}, r, l}=R^{3 / 2} \sum_{k}(1-R)^{k} \tilde{K}_{r, l, k, 0}^{r_{1}, l}\left(T_{1}\right) .
$$

Для степенных потенциалов $\tilde{K}_{r, l, k, 0}^{r_{1}, l}\left(T_{1}\right)=R^{\mu} K_{r, l, k, 0}^{r_{1}, l}$, где $K_{r, l, k, 0}^{r_{1}, l}$ - подмножество безразмерных нелинейных МЭ в стандартном базисе. Отметим, что в наших программах расчета стандартных линейных МЭ, основанных на рекуррентных соотношениях, обязательно рассчитываются и табулируются все нелинейные МЭ $K_{r, l, k, 0}^{r, l}$.

Расчет $\bar{\Lambda}_{r_{1}, r, l}\left(T_{1}\right)$ по формуле (10) значительно проще прямого численного интегрирования и позволяет считать $\bar{\Lambda}$ при сколь угодно больших индексах.

Для любых моделей с постоянной частотой столкновений выражение (10) упрощается, поскольку МЭ отличны от нуля только, если $k=r-r_{1}$ :

$$
\bar{\Lambda}_{r, r_{1}, l}=R^{3 / 2}(1-R)^{r-r_{1}} K_{r_{1}, l, r-r_{1}, 0}^{r, l} .
$$

В случае резонансной перезарядки с постоянной частотой столкновений матричные элементы $K_{r_{1}, l, k, 0}^{r, l}$ для произвольного $k$ принимают вид

$$
K_{r_{1}, l, k, 0}^{r, l}=R^{3 / 2}\left(\delta_{l 0} \delta_{k r} \delta_{r_{1} 0}-\delta_{r_{1} r} \delta_{k 0}\right) .
$$

Ранее в [16] рассматривался переход от разложения $\Phi Р$ в базисе с температурой весового максвеллиана $T_{n}$ и величиной средней скорости $u_{n}$ к базису с соответствующими параметрами $\bar{T}_{n}, \bar{u}_{n}$ и с коэффициентами разложения соответственно $C_{r, l}^{n}$ и $\bar{C}_{r_{1}, l_{1}}^{n}$. Векторы $\mathbf{C}^{\mathbf{n}}$ и $\overline{\mathbf{C}}^{\mathbf{n}}$ при переходе от базиса $W=\left(T_{n}, u_{n}\right)$ к базису $\bar{W}=\left(\bar{T}_{n}, \bar{u}_{n}\right)$ связаны соотношением

$$
\bar{C}_{r_{1}, l_{1}}^{n}=\sum_{r, l}^{\infty} D_{r_{1}, l_{1}, r, l}(\bar{W}, W) C_{r, l}^{n} .
$$

Компоненты матрицы перехода $D$ определяются как скалярное произведение

$$
D_{r_{1}, l_{1}, r, l}(\bar{W}, W)=\frac{1}{g_{r_{1}, l}} \int H_{r_{1}, l_{1}}\left(\mathbf{c}_{1}\right) M(W) H_{r, l}(\mathbf{c}) d^{3} c .
$$

Интеграл (12) вычислен в [16]. В настоящей работе мы имеем дело с частным случаем, когда $l_{1}=l$ и $u_{n}=\bar{u}_{n}=0$. Тогда компоненты матрицы $D$ имеют следующий простой вид:

$$
D_{r_{1}, r, l}=\frac{r_{1} ! R_{i}^{r_{1}+l / 2}\left(1-R_{n}\right)^{r_{1}-r}}{r !\left(r_{1}-r\right) !}, \quad R_{n}=T_{n} / \bar{T}_{n} .
$$

Предлагается на каждом временном промежутке $\Delta \tilde{t}$ температуру нового базиса $T_{b}$ определять по формуле

$$
T_{b} / T_{0}=1+(a-1)\left(1-e^{-b \tilde{t}}\right) .
$$

Здесь параметры $a$ и $b$ выбираются согласованно с зависимостью энергии ионов от времени. Был проведен 


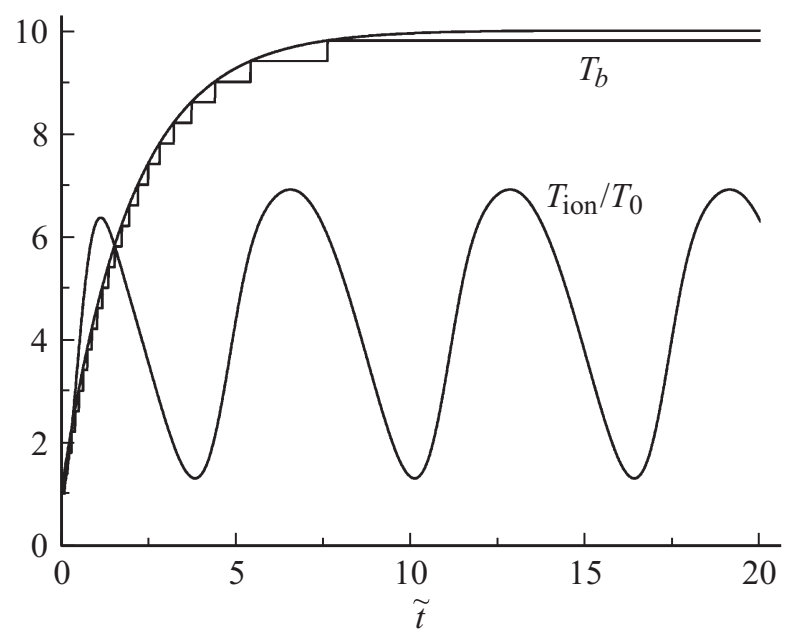

Рис. 1. Выбор температуры базиса при $\varepsilon_{0}=50, \omega=0.5$.

анализ оптимальных значений $a$ и $b$. Оказалось, что оптимальное значение $a$ примерно в $1.5-2$ раза превышает максимальную температуру ионов, непрерывно меняющуюся в ходе колебательного процесса по закону

$$
T_{\text {ion }} / T_{0}=1-C_{1,0}(\tilde{t}) .
$$

Оптимальное значение $b$ при умеренных $\alpha$ оказалось близким к 1/3, т.е. время релаксации температуры базиса примерно в 3 раза больше $\tau_{\beta}$. При больших $\alpha$ этот параметр подбирался отдельно для каждого $\alpha$.

Задача решалась методом Рунге-Кутты 4-го порядка. При переходе к новому промежутку времени проводился пересчет моментов по формуле (11).

Выбор температуры (14) позволил переходить к новому базису не на каждом временно́м шаге, а только в ограниченном числе точек. Это сократило время расчета на персональном компьютере на 2 порядка при той же точности расчетов. На рис. 1 приведен пример зависимости от времени непрерывно меняющейся температуры базиса (14), температуры базиса, изменяющейся скачкообразно, и температуры ионов (15).

Функция распределения строилась по формуле (6). При этом мы полагали $L_{0}=N_{0}=128$. Переход от модифицированных моментов $\overline{\mathbf{C}}^{\mathbf{n}}$ к стандартным $\mathbf{C}^{\mathbf{n}}$ проводился по формуле, обратной (11), с $R_{n}=\bar{T}_{n} / T_{0}$.

Отладка численной схемы проводилась с помощью сравнения решения для СЕМ-модели с точным аналитическим решением [22].

В заключение этого раздела напомним, как выражаются приведенные физические моменты, плотность тока и энергия ионов через стандартные моменты [20]. Так, плотность приведенного электрического тока равна

$$
\tilde{j}=\frac{j}{\alpha}=\frac{C_{0,1}}{2 \alpha},
$$

приведенные поправки к равновесным значениям поперечной и продольной энергий ионов имеют вид

$$
w_{t}=\frac{-C_{1,0}-0.5 C_{0,2}}{\alpha^{2}}, \quad w_{l}=\frac{C_{0,2}-C_{1,0}}{2 \alpha^{2}} .
$$

Приведенная поправка к полной энергии имеет вид

$$
w=w_{t}+w_{l}=-\frac{3 C_{1,0}}{2 \alpha^{2}} .
$$

\section{Режим малых $\alpha(\alpha \ll 1)$}

При изучении подвижности в постоянном внешнем электрическом поле выяснилось $[12,18]$, что параметром малости является безразмерный параметр $\epsilon$, т.е. для всех сечений при $\epsilon \ll 1$ подвижность $K=$ const. Наши исследования тока и энергии ионов [20] показали, что в переменном электрическом поле при $\alpha \ll 1$ для $\tilde{j}$ и $w$ получаются следующие аналитические выражения:

$$
\begin{gathered}
\tilde{j}=\tilde{j}_{a}+\tilde{j}_{p}, \quad \tilde{j}_{a}(\tilde{t})=-\frac{\omega \exp (-\tilde{t})}{1+\omega^{2}}, \\
\tilde{j}_{p}(\tilde{t})=A^{j} \cos \left(\omega \tilde{t}+\Delta_{E}^{j}\right), \quad A^{j}=\frac{\omega}{\sqrt{1+\omega^{2}}}, \\
\Delta_{E}^{j}=-\operatorname{arctg}(\omega) .
\end{gathered}
$$

Здесь $A^{j}-$ амплитуда плотности электрического тока ионов, а $\Delta_{E}^{j}$ - его фазовый сдвиг относительно электрического поля. Индексы $p$ и $а$ относятся соответственно к периодической и апериодической частям зависимости плотности тока от времени.

Для поправки к полной энергии имеем

$$
\begin{gathered}
w_{a}=-2 e^{-\tilde{t}}\left(\frac{\omega^{2}}{1+4 \omega^{2}}+\sin (\omega \tilde{t}) \frac{\omega^{2}}{1+\omega^{2}}\right), \\
w_{p}=a_{0}+A^{w} \cos \left(2 \omega \tilde{t}+\Delta_{E}^{w}\right), \quad a_{0}=\frac{\omega^{2}}{1+\omega^{2}} .
\end{gathered}
$$

Здесь $a_{0}-$ среднее значение периодической части полной энергии. Амплитуда $A^{w}$ имеет вид

$$
A^{w}=\frac{\omega^{2}}{\sqrt{\left(1+4 \omega^{2}\right)\left(\omega^{2}+1\right)}} .
$$

Для фазового сдвига относительно поля $\Delta_{E}^{w}$ имеем

$$
\Delta_{E}^{w}= \begin{cases}\operatorname{arctg} \frac{3 \omega^{2}}{\left(2 \omega^{2}-1\right)}, & \omega \leq \sqrt{0.5} \\ \operatorname{arctg} \frac{3 \omega^{2}}{\left(2 \omega^{2}-1\right)}-\pi, & \omega>\sqrt{0.5}\end{cases}
$$

Видно, что в режиме малых $\alpha$ вся зависимость тока и полной энергии от сечения взаимодействия частиц определяется величиной $\tau_{\beta}$.

Было проведено сравнение численных расчетов ФР с точностью до второго приближения по $\alpha$ для различных сечений с точным аналитическим решением для CЕМмодели [22]. В первом приближении имеется полное совпадение в пределе достаточно высоких частот. Незначительное отличие во втором приближении даже при очень высоких частотах связано с генерацией поперечной энергии для любых сечений рассеяния, кроме резонансной перезарядки. 
Таблица 2. Коэффициенты $C_{0,1}$ и $C_{1,0}$ для различных моделей взаимодействия. $\omega=2$

\begin{tabular}{c|c|c|c|c|c|c|c}
\hline \multirow{2}{*}{ Модель } & \multicolumn{3}{|c|}{$C_{0.1}$} & \multicolumn{4}{c}{$C_{1.0}$} \\
\cline { 2 - 8 } & $\alpha=0.1$ & $\alpha=1$ & $\alpha=10$ & $\alpha=0.1$ & $\alpha=0.5$ & $\alpha=1$ & $\alpha=10$ \\
\hline HS & 0.0178 & 1.74 & 10.4 & -0.000812 & -0.202 & -0.786 & -29.3 \\
\hline ANHS & 0.0178 & 1.74 & 10.5 & -0.000821 & -0.202 & -0.790 & -30.6 \\
\hline CЕНS & 0.0177 & 1.73 & 10.0 & -0.000809 & -0.201 & -0.779 & -25.7 \\
\hline CЕM & 0.0179 & 1.79 & 17.9 & -0.000823 & -0.204 & -0.823 & -82.3 \\
\hline ММ & 0.0178 & 1.78 & 17.9 & -0.000823 & -0.204 & -0.823 & -82.3 \\
\hline РММ & 0.0178 & 1.78 & 17.8 & -0.000823 & -0.204 & -0.823 & -82.3
\end{tabular}

Расчеты для различных сечений показали, что при малых $\alpha$ с ростом частоты все моменты, кроме $C_{0,0}, C_{0,1}$, $C_{1,0}$ и $C_{0,2}$ убывают быстрее, чем $\alpha^{2}$.

В [20] с использованием только этих моментов получено следующее аналитическое выражение для периодической части ФР:

$$
\begin{aligned}
f_{p}(\mathbf{c}, \tilde{t})= & M(c)\left(1+2 \alpha c_{z} \tilde{j_{p}}+\alpha^{2}\left(2 c_{z}^{2}-1\right) w_{p}\right. \\
& \left.-2 \alpha^{2} c^{2} P_{2}(\cos \Theta) w_{t}\right)
\end{aligned}
$$

где $\tilde{j_{p}}$ и $w_{p}$ определяются формулами (20) и (22). Функция $P_{2}(x)$ - это второй полином Лежандра, а $w_{t}-$ поправка к поперечной энергии ионов (17). В случае резонансной перезарядки $w_{t}=0$.

\section{Результаты расчетов}

В ходе исследования для всех рассматриваемых моделей при произвольных $\alpha$ были изучены как амплитуды колебаний и сдвиги фаз тока и энергии ионов, так и их функции распределения. Рассматривалась только периодическая часть процесса. Все расчеты проводились в $\beta$-нормировке.

На рис. 2, а представлены амплитуды приведенного тока $\tilde{j}(\alpha)$ для широкого диапазона частот поля и трех моделей взаимодействия. Видно, что в случае HS- и CEHS-моделей для каждой из рассмотренных частот имеется область значений $0 \leq \alpha \leq 1$, в которой амплитуда почти постоянна и фактически совпадает с соответствующей зависимостью для СЕМ-модели. Подчеркнем, что с ростом частоты ширина этой области растет и при $\omega=50$ амплитуда не зависит от $\alpha$ вплоть до $\alpha \sim 10$. При дальнейшем увеличении $\alpha$ наблюдается уменьшение амплитуды приведенного тока и при $\alpha \rightarrow \infty$ она стремится к нулю.

Сдвиг фаз между током и полем приведен на рис. 2, $b$. Так же как и в случае амплитуды, для каждой частоты есть область значений $\alpha$, где сдвиг фаз изменяется мало. С ростом значений $\alpha$ сдвиг фаз начинает убывать по модулю и в пределе больших $\alpha$ стремится к нулю.
Из рис. 2 следует, что в $\beta$-нормировке для всех значений $\alpha$ при любых частотах величина плотности электрического тока полностью определяется зависимостью сечения рассеяния от относительной скорости и совсем не зависит или зависит весьма незначительно от
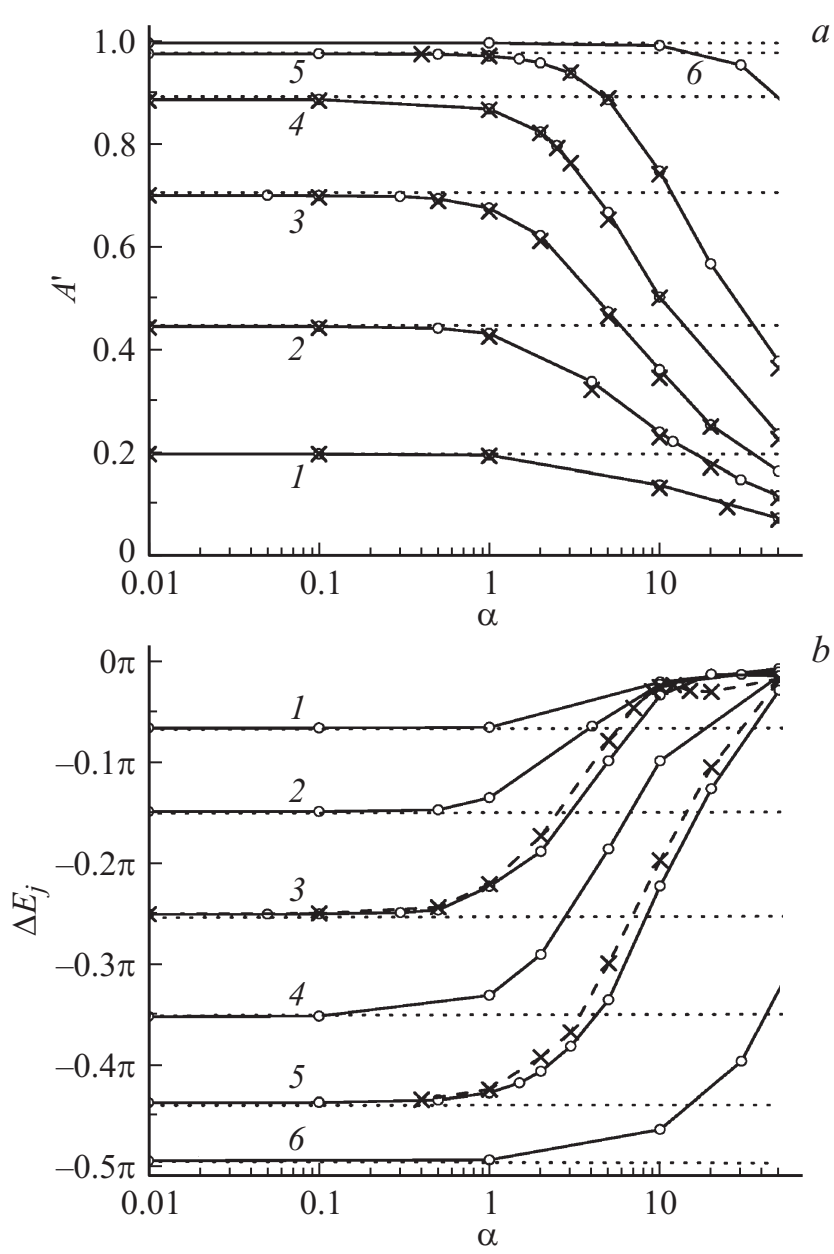

Рис. 2. Зависимости амплитуды $A^{j}(a)$ и сдвига фаз $\Delta_{E}^{j}(b)$ плотности приведенного тока от $\alpha$ для различных частот $\omega$ : $1-0.2,2-0.5,3-1,4-2,5-5,6-50$. Сплошная линия и кружки соответствуют HS-модели, штриховая и крестики - CEHS-модели, а пунктир - CEM-модели. 


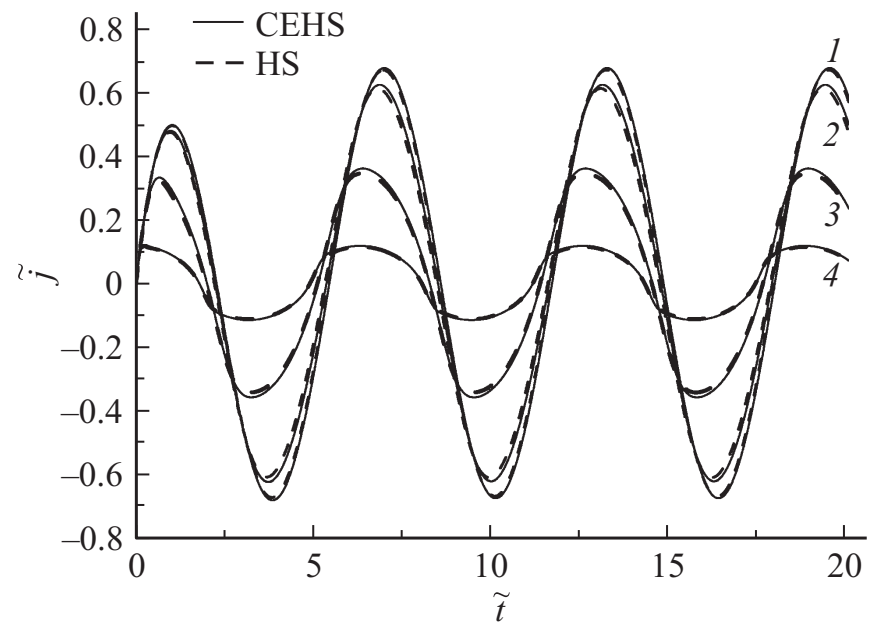

Рис. 3. Зависимость плотности приведенного тока $j(t)$ для $\omega=1$ и различных значений $\alpha: 10.1-1,2-2,3-10$, 4 - 100. Сплошная кривая - HS-модели, пунктир - CEHSмодели.

углового рассеяния. Анализ наших расчетов для ANHS-, MМ- и РММ-моделей показал, что все выводы, следующие из рис. 2, сохраняются для этих моделей с еще большей точностью. В качестве иллюстрации в табл. 2 приведен момент $C_{0,1}$ во времена, соответствующие максимальному значению плотности тока при частоте $\omega=2$ для $\alpha=0.1,1$ и 10.

Таким образом, для плотности тока режим малых $\alpha$ $(\alpha<0.01)$ сохраняется вплоть до $\alpha=1$ для всех частот и для всех рассматриваемых нами сечений, причем эта область растет с ростом частоты.

На рис. 3 представлена плотность $\tilde{j}(\tilde{t})$ при различных $\alpha$. Видно, что при $\alpha \gg 1$ временна́я зависимость плотности тока начинает отличаться от гармонической, оставаясь периодической с периодом, равным периоду поля.

Из результатов расчетов видно, что плотности тока для $\tau$ - и $\lambda$-моделей различаются, в то время как внутри каждой группы моделей они почти совпадают. Следовательно, поведение приведенного электрического тока определяется в основном полным сечением рассеяния, причем при $\alpha \leq 1$ наблюдается подобие зависимостей (20) для всех рассмотренных сечений с коэффициентом подобия, равным $\tau_{\beta}$.

Помимо тока важной характеристикой процесса является энергия ионов. В отличие от тока зависимости полной энергии ионов от времени почти совпадают для всех частот только при $\alpha<0.5$ (см. табл. 2) и эта область увеличивается с ростом частоты поля. В этой области, так же как и в случае с током, для всех моделей можно использовать аналитические формулы (22), полученные для СЕМ-модели. Однако с ростом величины параметра $\alpha$ появляется заметное различие в поведении энергии даже внутри группы $\lambda$-моделей. Сказанное выше хорошо иллюстрируется рис. 4 , где для случая $\lambda$-моделей приведены зависимости полной энергии от времени при различных значениях $\alpha$. Обратим внимание, что для $\tau$-моделей при любых $\alpha$ остаются справедливыми аналитические зависимости (22).

Помимо резонансной перезарядки для других моделей взаимодействия существует отличная от нуля поправка к поперечной энергии ионов. В отличие от поправки к полной энергии поправка к поперечной энергии сильно зависит от угловой части сечения рассеяния даже при малых $\alpha$. Это хорошо видно из рис. 5. Отметим, что с ростом параметра $\alpha$ эта разница увеличивается. Кроме того, с ростом частоты амплитуда колебаний накапливаемой поперечной энергии стремится к нулю, a eе среднее значение увеличивается и выходит на насыщение.

Теперь рассмотрим функцию распределения. Именно по ней контролировалось установление периодичности процесса. Наши расчеты показали, что физические моменты сохраняют поведение, соответствующее режиму малых $\alpha$, значительно дольше, чем функция распределения.
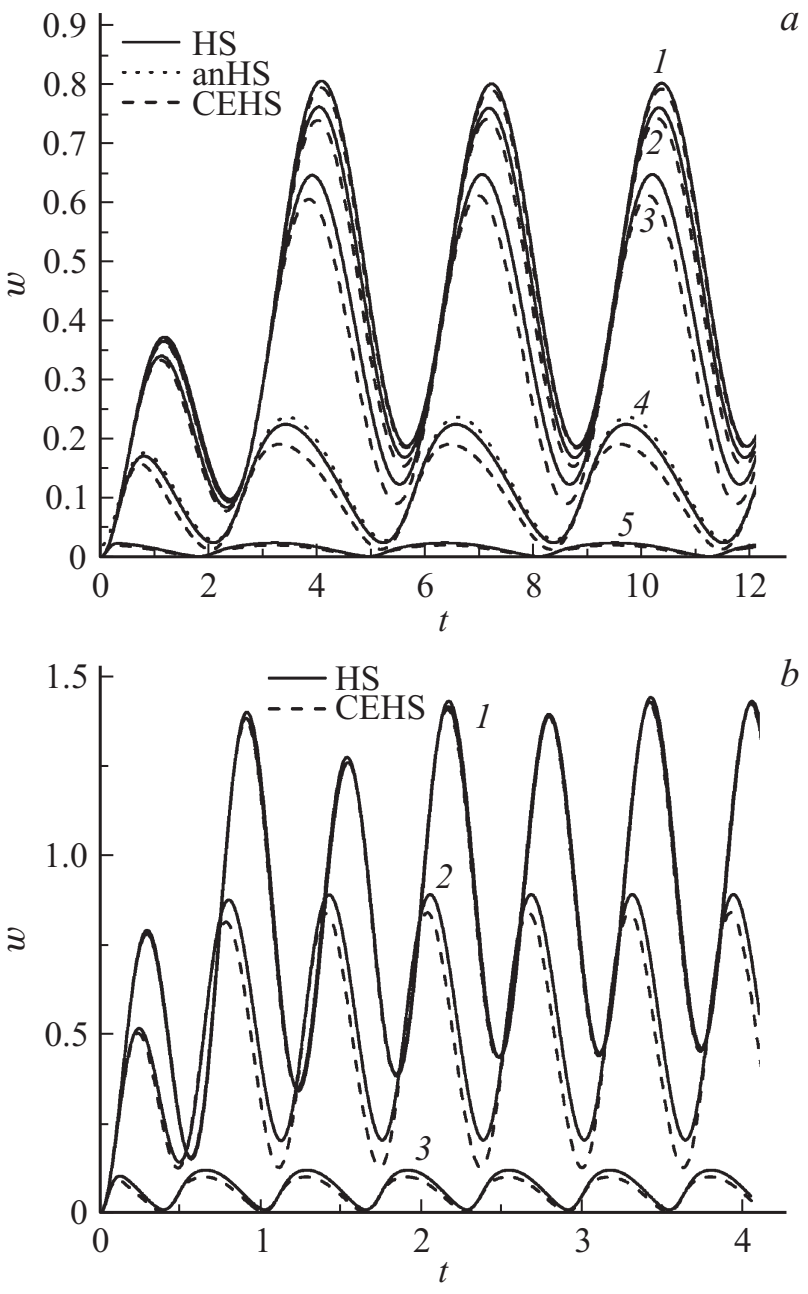

Рис. 4. Зависимость поправки к полной энергии от времени. (a) $\omega=1: \alpha=0.5$ (1), $\alpha=1$ (2), $\alpha=2$ (3), $\alpha=10$ (4), $\alpha=100$ (5); (b) $\omega=5: \alpha=0.5$ (1), $\alpha=10$ (2), $\alpha=100$ (3). 
На рис. 6 представлено первое приближение по $\alpha$ поправки $\Phi_{p}^{(1)}\left(c_{z}\right)$ к периодической части $\Phi \mathrm{P} f_{p}$, отнесенное к $\tilde{j}_{p}$, для HS-модели для трех величин $\alpha$ и $c_{\rho}=0$.
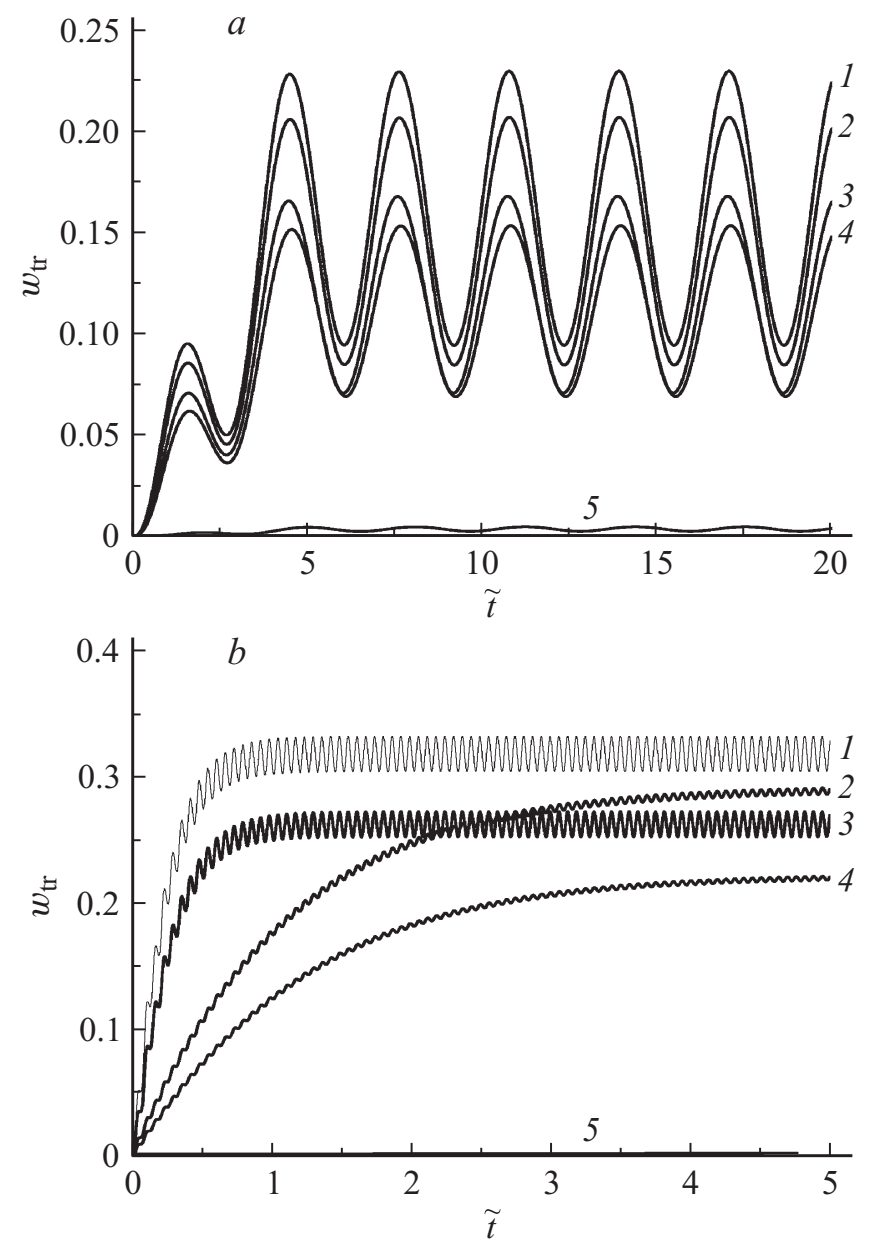

Рис. 5. Поправка к поперечной энергии ионов $w_{\operatorname{tr}}(t)$. (a) $\alpha=1, \omega=1$. (b) $\alpha=10, \omega=50$. Кривая 1 - ANHS, 2 - МM, 3 - HS, 4 - РММ и 5 - СЕМ-, СЕHS-модели.

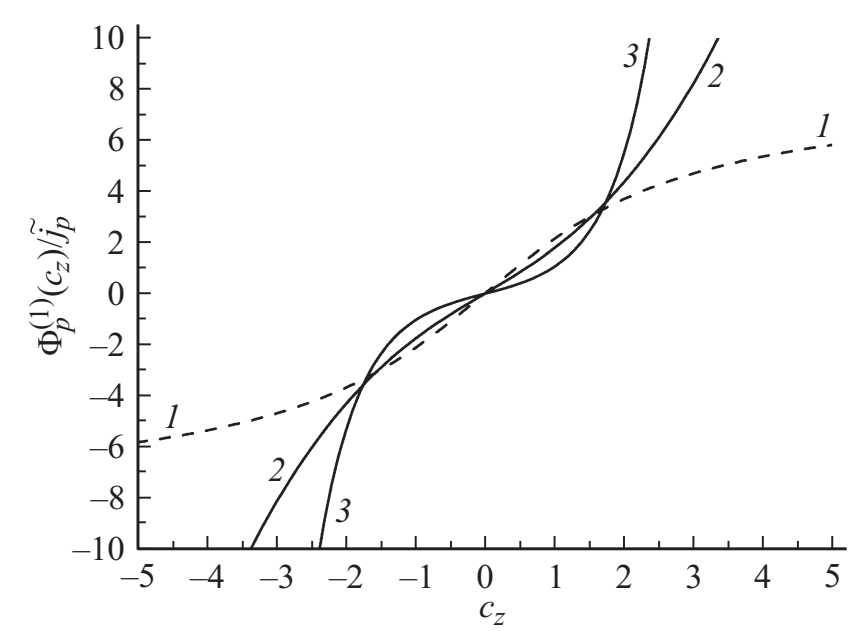

Рис. 6. Первое приближение поправки к функции распределения $\Phi_{p}^{1}\left(c_{z}\right) / j_{p}$. HS-модель, $\omega=1$. Кривая $1-\varepsilon_{0}=0.1$, $2-\varepsilon_{0}=0.5,3-\varepsilon_{0}=1$.
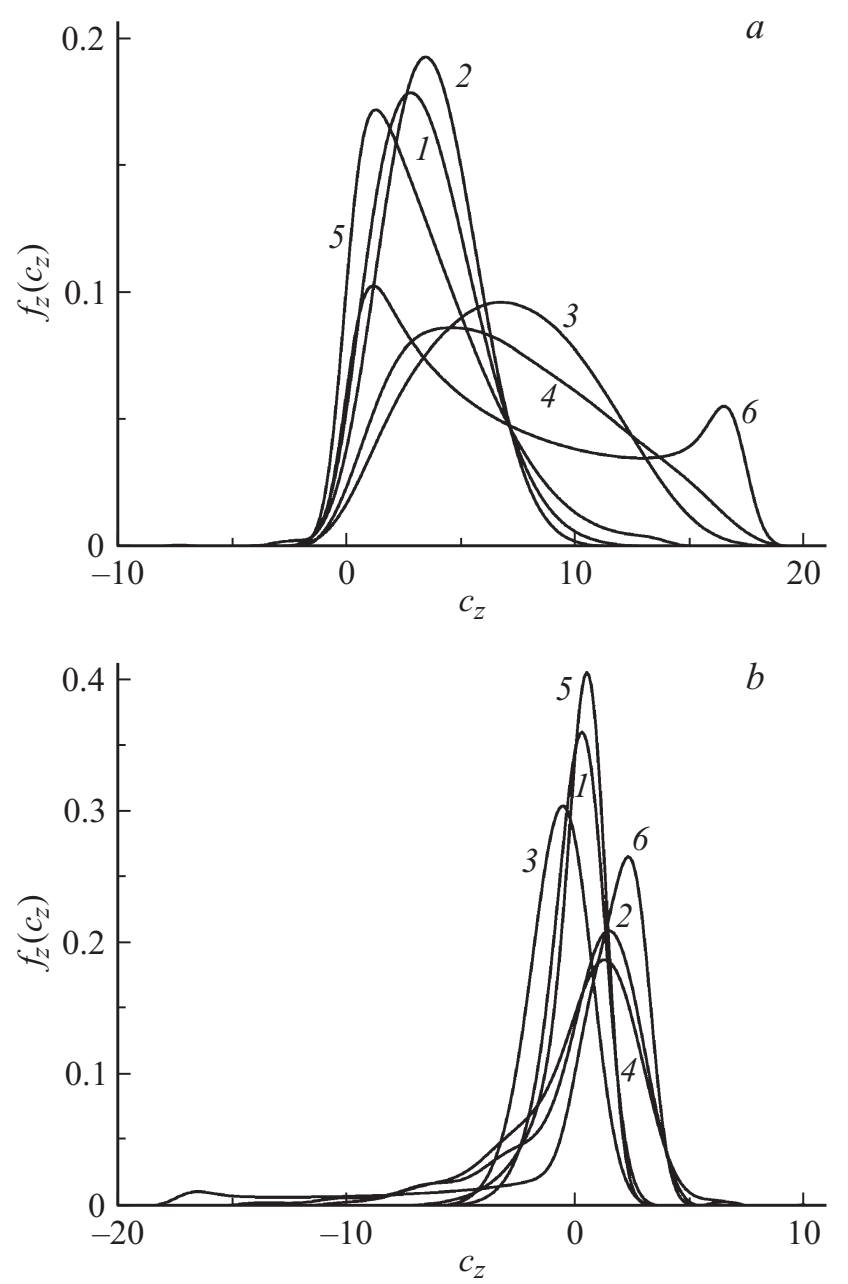

Рис. 7. Функция распределения $f_{z}\left(c_{z}\right)$ в направлении поля для различных моделей взаимодействия при $(a)$ максимальном токе и $(b)-$ минимальном. $\alpha=10, \omega=1$. Кривая $1-\mathrm{HS}$, 2 - anHS, 3 - MM, 4 - PMM, 5 - CEHS, 6 - СЕМ-модели.

Видно значительное отклонение $\Phi_{p}^{(1)}\left(c_{z}\right)$ от прямых, соответствующих режиму малых $\alpha[20]$. Это связано с существенным влиянием более высоких моментов $C_{r, 1}$ при $r>0$.

Развитый нами модифицированный моментный метод позволил вычислять функцию распределения при произвольных значениях параметра $\alpha$. Интересно сравнить поведение функции распределения в направлении поля для различных моделей взаимодействия. На рис. 7 приведены зависимости проинтегрированной по $c_{\rho}$ функции распределения ионов $f_{z}\left(c_{z}\right)$ в моменты максимального и нулевого тока.

Как можно видеть из рисунков, функция распределения сильно отличается от равновесной даже при нулевом токе. Особенно хочется подчеркнуть, что даже при скоростях, больших 10 тепловых, имеется достаточно большое число частиц. Кроме того, наблюдается сильная зависимость от распределения сечения рассеяния по углам. Для моделей с постоянной длиной свободного 
пробега характерна локализация ФР в более узком интервале скоростей.

\section{Заключение}

Разработан и успешно применен к задаче о движении ионов в собственном газе в гармоническом электрическом поле модифицированный моментный метод. В отличие от стандартного моментного метода этот метод позволил проводить расчеты при произвольных параметрах поля. Это стало возможно благодаря использованию переменной температуры базиса и разработанной нами ранее процедуре расчета линейных МЭ интеграла столкновений через затабулированные нами ранее стандартные нелинейные МЭ.

Были проведены расчеты тока и энергии при различных значениях параметра $\alpha=e E_{0} /\left(m \Omega v_{T}\right)$. Оказалось, что аналитические формулы для тока и энергии, полученные в [20], остаются справедливыми вплоть до $\alpha=1$ для тока и $\alpha=0.5$ для энергии. Таким образом, если

$$
e E_{0} / m<\Omega v_{T}
$$

то в размерных единицах для тока $J=j v_{T}$ имеем следующее аналитическое выражение:

$$
\begin{gathered}
J(t)=J_{a}(t)+J_{p}(t), \\
J_{a}(t)=-\frac{e E_{0} \beta \tau}{m} \frac{\exp (-t / \beta \tau)}{1+(\beta \tau \Omega)^{2}}, \\
J_{p}(t)=\frac{e E_{0} \beta \tau}{m} A^{j} \cos \left(\Omega t+\Delta_{E}^{j}\right), \\
A^{j}=\frac{1}{\sqrt{1+(\beta \tau \Omega)^{2}}}, \quad \Delta_{E}^{j}=-\operatorname{arctg}(\beta \tau \Omega) .
\end{gathered}
$$

Аналогичные формулы для полной энергии в размерном виде можно выписать, используя (22)-(24).

Неопределенным в этих формулах оказывается стандартное время релаксации. Например, для модели твердых шаров $\tau=1 /\left(n \sigma_{0} v_{T}\right)$. Эта величина должна определяться либо из экспериментов по определению подвижности при малых постоянных полях, либо так, как было описано в [20] по определению $\tau_{e f f}=\tau_{\beta}$.

Проведенные нами исследования показали, что поправка к поперечной энергии значительно сильнее, чем другие физические моменты, зависит от углового распределения сечения взаимодействия.

Помимо физических моментов модифицированный моментный метод позволил нам впервые строить с большой точностью функцию распределения вплоть до больших скоростей. Расчеты показали сильную зависимость вида ФР как от энергетической, так и от угловой частей сечения рассеяния.

В настоящей работе мы рассмотрели только случай равных масс частиц. Ближайшей целью нашей дальнейшей работы будет обобщение этих результатов на случай частиц разной массы.
Один из соавторов (Герасименко А.Б.) благодарит РФФИ (грант № 15-08-03440) за частичную поддержку.

\section{Список литературы}

[1] Каган Ю.М., Перель В.И. // ДАН СССР. 1954. Vol. 98. C. 575-578.

[2] Wannier G.H. // Bell. Syst. Tech. J. 1953. Vol. 32. P. 170-254.

[3] Сена Л. // ЖЭТФ. 1946. Вып. 8. С. 734-738.

[4] Мак-Даниэль И., Мэзон Д. Подвижность и диффузия ионов в газах. М.: Мир, 1976. 422 с.

[5] Maneschijn A., Goedheer W.J. // J. Appl. Phys. 1991. N 69. P. 2923.

[6] Liberman M.A., Lichtenberg A.J. Principles of Plasma Discharge and Material Processing. Hoboken, NJ: Wiley Interscience, 2005. $800 \mathrm{p}$.

[7] Ness K.F. // Aust. J. Phys. 1995 Vol. 48. P. 557-569.

[8] White R.D., Ness K.F., Robson R.E., Li B. // Phys. Rev. E. 1999. Vol. 60. P. 2231-2249.

[9] Sugawara H., Tagashira H., Sakay Y. // J. Phys. D: Appl. Phys. 1996. N 29. P. 1168-1174.

[10] Dujko S., White R.D., Petrovic Z.Lj., Robson R.E. // Phys. Rev. E. 2010. Vol. 81. P. 046403.

[11] Viehland L.A., Maeson E.A. // Ann. Phys. 1978. Vol. 110. P. 287-328.

[12] Mason E.A., McDaniel E.W. Transport Properties of Ions in Gases. NY: Wiley, 1988. 489 p.

[13] Viehland L.A., Siems W.F. // J. Am. Soc. Mass. Spectrom. 2012. N 23. Vol. 11. P. 1841-1854.

[14] Kumar K. // Ann. Phys. 1966. Vol. 37. P. 113-141.

[15] White R.D., Ness K.F., Robson R.E. // Appl. Surf. Sci. 2002. Vol. 192. P. 26-49.

[16] Эндер А.Я., Эндер И.А. Интеграл столкновений уравнения Больцмана и моментный метод. СПб: Изд-во СПбГУ, 2003. $224 \mathrm{c}$.

[17] Ender A.Ya., Ender I.A. // Transport Theory and Stat. Phys. 2007. Vol. 56. P. 563-588.

[18] Ender A.Y., Ender I.A., Gerasimenko A.B. // The Open Plasma Phys. J. 2009. Vol. 2. P. 24-62.

[19] Эндер А.Я., Эндер И.А., Герасименко А.Б. // ЖТФ. 2010. Т. 80. Вып. 2. С. 18-28.

[20] Эндер А.Я., Эндер И.А., Герасименко А.Б. // ЖТФ. 2015. Т. 85. Вып. 1. С. 15-25.

[21] Ферцигер Джс., Капер Г. Математическая теория процессов переноса в газах. М.: Мир, 1976. 557 с.

[22] Эндер А.Я., Эндер И.А., Герасименко А.Б. // ЖТФ. 2013. т. 83. Вып. 7. С. 6-15. 